\title{
Understanding the Interactions between Porphyrin-Containing Photosensitizers and Polymer-Coated Nanoparticles in Model Biological Environments
}

\author{
Samir V. Jenkins, ${ }^{\dagger}$ Avinash Srivatsan, ${ }^{\dagger}$ Kimberly Y. Reynolds, ${ }^{\dagger}$ Feng Gao,${ }^{\dagger}$ Yongbin Zhang, ${ }^{\S}$ \\ Colin D. Heyes, ${ }^{\dagger}$ Ravindra K. Pandey, ${ }^{\ddagger}$ Jingyi Chen ${ }^{\dagger, *}$ \\ †Department of Chemistry and Biochemistry, University of Arkansas, Fayetteville, AR 72701 \\ ${ }^{\ddagger}$ Photodynamic Therapy Center, Roswell Park Cancer Institute, Buffalo, NY 14263 \\ ${ }^{\S}$ NCTR/ORA Nanotechnology Core Facility, National Center for Toxicological Research, U.S. \\ Food and Drug Administration, Jefferson, AR 72079 \\ *Corresponding author: chenj@uark.edu
}




\begin{abstract}
:
Non-covalent incorporation of hydrophobic drugs into polymeric systems is a commonly-used strategy for drug delivery because non-covalent interactions minimize modification of the drug molecules whose efficacy is retained upon release. The behaviors of the drug-polymer delivery system in the biological environments it encounters will affect the efficacy of treatment. In this report, we have investigated the interaction between a hydrophobic drug and its encapsulating polymer in model biological environment using a photosensitizer encapsulated in a polymercoated nanoparticle system. The photosensitizer, 3-(1'-hexyloxyethyl)-3devinylpyropheophorbide-a (HPPH), was non-covalently incorporated to the poly(ethylene glycol) (PEG) layer coated on Au nanocages (AuNCs) to yield AuNC-HPPH complexes. The non-covalent binding was characterized by Scatchard analysis, fluorescence lifetime, and Raman experiments. The dissociation constant $\left(K_{d}\right)$ between PEG and HPPH was found to be $\sim 35 \mu \mathrm{M}$ with a maximum loading of $\sim 2.5 \times 10^{5} \mathrm{HPPH} / \mathrm{AuNC}$. The release was studied in serum mimetic environment and in vesicles that model human cell membranes. The rate of protein-mediated drug release decreased when using a negatively-charged or cross-linked terminus of the surfacemodified PEG. Furthermore, the photothermal effect of AuNCs can initiate burst release, and thus allow control of the release kinetics, demonstrating on-demand drug release. This study provides insights regarding the actions and release kinetics of non-covalent drug delivery systems in biological environments.
\end{abstract}

Keywords: gold nanostructure, drug delivery, PEG coating, controlled release 


\section{Introduction}

Controlled release systems for drug delivery using nanocarriers have been developed and studied for more than three decades. ${ }^{1}$ Au nanostructures have been used as drug delivery vehicles in chemotherapy because of their biocompatibility, facile surface modification, and robust optical properties. $^{2-8}$ While drug molecules can be covalently immobilized on the nanoparticles' surface, ${ }^{2,8-10}$ non-covalent interactions are particularly appealing because they minimize modification of the drug molecules, whose efficacy is then largely retained upon release. Several strategies have been developed to non-covalently tether molecules through electrostatic and hydrophobic interactions to polymer-coated nanoparticles, which include wrapping into layer-bylayer assembled polyelectrolytes, ${ }^{11}$ entrapping in a polymer monolayer, ${ }^{12-14}$ encapsulating into a phospholipid bilayer, ${ }^{15}$ and absorbing into a hydrogel. ${ }^{16}$ Among these methods, poly(ethylene glycol) (PEG) coated nanoparticles are advantageous because PEG used widely in the pharmaceutical industry is generally nontoxic and possesses antifouling properties well-suited for increasing circulation half-life and minimizing immunological clearance. ${ }^{17}$ The graft density of PEG was found to significantly affect the biological fate of the particles. ${ }^{18}$ Hydrophobic phthalocyanine photosensitizers ${ }^{19-21}$ or anti-cancer drugs $s^{22,23}$ have been delivered within the surface PEG layer or inside the hydrophobic pocket for photodynamic therapy or chemotherapy. We recently applied a similar system to deliver a hydrophobic porphyrin-derivative (3-(1'hexyloxyethyl)-3-devinylpyropheophorbide-a, HPPH, structure shown in Fig. 1A) for imageguided photodynamic therapy with enhanced efficacy. ${ }^{14}$

In this work, we systematically investigate the binding affinity and the release kinetics of HPPH from PEG-coated Au nanocages (AuNCs) to further elucidate the behavior of the drugpolymer delivery system. The induced-dipole/induced-dipole interactions between the 
pheophorbide and the PEG backbone serve as the driving force to load molecules into the PEG layer. ${ }^{24} \mathrm{HPPH}$ is stably integrated within the PEG coating of AuNC-HPPH in saline solution. The release itself is mediated by either serum proteins (specifically albumin in our model) or cell membranes (modelled here with vesicles). The serum proteins unloaded the drug over several hours and the release of entrapped molecules can be endogenously controlled by the charge and functionality of the PEG terminus. Additionally, the release rate can be exogenously controlled by utilizing the photothermal (PT) effect of $\mathrm{Au}$ nanostructures which allows external and temporal control of the drug release by non-invasive near-infrared light. ${ }^{25-27,11,28}$ By manipulating these variables, we are able to demonstrate on-demand unloading of the drug with minimal premature loss and were able to suggest the relative affinity of the drug for soluble proteins versus cell membranes.

\section{METHODS}

Incorporation of HPPH to AuNC-PEG to form AuNC-HPPH complexes. HPPH (7 mg, $11 \mu \mathrm{mol})$ was dissolved in $2.5 \mathrm{~mL}$ DMSO at a concentration of $4.5 \mathrm{mM}$ as stock solution. This stock solution $(0.25 \mathrm{~mL})$ was diluted in $4 \mathrm{~mL}$ of phosphate buffered saline (PBS) via dropwise addition in an ice bath to a concentration of $\sim 183 \mu \mathrm{M}$. The diluted HPPH solution was flushed with Ar for 5 min and allowed to mix for 5 additional min. The AuNC-PEG suspension $(3 \mathrm{nM}, 2 \mathrm{~mL})$ was added dropwise to the HPPH solution. The reaction was allowed to stir overnight under the protection of $\mathrm{Ar}$ in the dark. The product containing non-covalently incorporated HPPH on AuNC-PEG was then washed with three cycles of centrifugation $\left(14,000 \mathrm{RPM}, 15 \mathrm{~min}, 20{ }^{\circ} \mathrm{C}\right)$ and redispersed in PBS with a final AuNC concentration of $6 \mathrm{nM}$. The loaded HPPH can be fully released from complexes by methanol. 
Cross-linking of AuNC-HPPH complexes. AuNC-PEG-NH ${ }_{3}{ }^{+}$was incorporated with HPPH to a concentration of $\sim 2.5 \times 10^{4} \mathrm{HPPH} / \mathrm{AuNC}$ using the above procedure. $\mathrm{PEG}_{250}$ (PEG with M.W. $=250)$, a diacidic cross-linker, was dispersed in borate buffer $(\mathrm{pH}=9)$ in an ice bath with stirring to a concentration of $2.0 \mathrm{mM}(4.2 \mu \mathrm{mol}, 1.0 \mathrm{mg})$. To this $2 \mathrm{~mL}$ solution, EDC (7.8 $\mu \mathrm{mol}$, $1.2 \mathrm{mg})$ and NHS (10.4 $\mu \mathrm{mol}, 1.2 \mathrm{mg})$ were added. The solution was then flushed with Ar for 20 min. AuNC-HPPH solution $(0.2 \mathrm{~mL})$ was added dropwise into the solution. The reaction was allowed to stir overnight in the dark under the protection of Ar. The product was recovered by two centrifugation cycles $\left(14,000 \mathrm{RPM}, 15 \mathrm{~min}, 20^{\circ} \mathrm{C}\right)$ and redispersed in PBS.

Preparation of Lipid Vesicles. 1,2-dimyristoyl-sn-glycero-3-phosphocholine (DMPC, $75 \mu \mathrm{mol}$ ) and 1,2-dimyristoyl-sn-glycero-3-phospho-(1'-rac-glycerol) (DMPG, $25 \mu \mathrm{mol})$ were dissolved in $5 \mathrm{~mL}$ chloroform to yield a $20 \mathrm{mM}$ lipid solution. The solvent was removed under high vacuum overnight. The lipids were hydrated in $5 \mathrm{~mL}$ PBS with vigorous shaking for $2 \mathrm{~h}$, following by $2 \mathrm{~h}$ in the bath sonicator, until the solution was no longer turbid. Lipid vesicles were stored at $4{ }^{\circ} \mathrm{C}$ under Ar.

Study of HPPH release kinetics. Recovered fluorescence was used to monitor the release kinetics of HPPH from AuNC-HPPH complexes. Typically, AuNC-HPPH was diluted in PBS or BSA in PBS $(4 \% \mathrm{w} / \mathrm{w}, \sim 575 \mu \mathrm{M})$ at a final concentration of $0.1 \mathrm{nM}$ AuNC. Fluorescence measurements were acquired after the dilution at various intervals throughout a $32 \mathrm{~h}$ period using $\lambda_{\mathrm{ex}}=605 \mathrm{~nm}$ and $\lambda_{\mathrm{em}}=668 \mathrm{~nm}$. Samples were kept at room temperature, in the dark, without stirring or unnecessary agitation between measurements. At the end of the data acquisition, $10 \mu \mathrm{L}$ of $0.5 \mathrm{M}$ aqueous $\mathrm{KCN}$ was added to dissolve AuNCs and the fluorescence spectrum was acquired to determine the maximum (100\%) HPPH signal for the sample. PBS samples were monitored 
using supernatant absorbance at various times following centrifugation. After measurement, the pellet was redispersed in the original supernatant.

To test the PT effect on the release kinetics, an 808-nm diode laser (Power Technology) was used to irradiate the suspension of AuNC-HPPH complexes. The laser intensity was adjusted to

a power density of $1 \mathrm{~W} / \mathrm{cm}^{2}$ by changing the spot size to maintain the temperature at $\sim 46{ }^{\circ} \mathrm{C}$. The fluorescence measurement was taken at different time periods over the course of $2 \mathrm{~h}$ irradiation, and irradiation began within $10 \mathrm{~s}$ of introduction of sample into BSA. On-demand release was demonstrated using 5 min irradiation pulses followed by 10 min without irradiation.

\section{RESULTS AND DISCUSSION}

The AuNC-HPPH complexes were prepared according to our previously-reported method by covalently attaching HS-PEG-X to the nanoparticle surface and subsequently encapsulating HPPH into the PEG coating. ${ }^{14}$ AuNCs with an average exterior edge length of $53 \pm 8 \mathrm{~nm}$ and interior edge length of $41 \pm 6 \mathrm{~nm}$ measured from TEM were synthesized for this study. After their surface was coated with $\mathrm{HS}-\mathrm{PEG}-\mathrm{NH}_{3}{ }^{+}$, the LSPR of the AuNCs was centered at $801 \mathrm{~nm}$ (Fig. 1B), slightly red-shifted from the LSPR of the "bare" AuNCs at $798 \mathrm{~nm}$ (Fig. S1A). This LSPR maximum falls within the tissue transparent window and overlaps the $808 \mathrm{~nm}$ wavelength of the diode laser utilized for PT release. Figure 1C shows the spectral changes after loading; the LSPR of AuNCs was further red-shifted to $829 \mathrm{~nm}$, which is attributed to a change in the refractive index of the local surface environment. ${ }^{29}$ The peaks at $512,545,617$, and $669 \mathrm{~nm}$ are attributed to absorption by the HPPH molecules (Fig. S1B). The concentration of AuNCs was calculated by the metal concentration from flame atomic absorbance spectroscopy and size information from TEM analysis. The extinction coefficient of AuNCs was derived based on 
Beer's Law to be $1 \times 10^{10} \mathrm{~cm}^{-1} \mathrm{M}^{-1}$ (Fig. S2A). The fluorescence was quenched after loading due to static/contact quenching in the HPPH, nanometal surface energy transfer, incident light being absorbed by the AuNCs, or a combination thereof. ${ }^{30-33}$ After the AuNCs were digested by KCN, the fluorescence was recovered, and then quantified by comparison to the calibration curve (Figs. 1D, S2B).

A
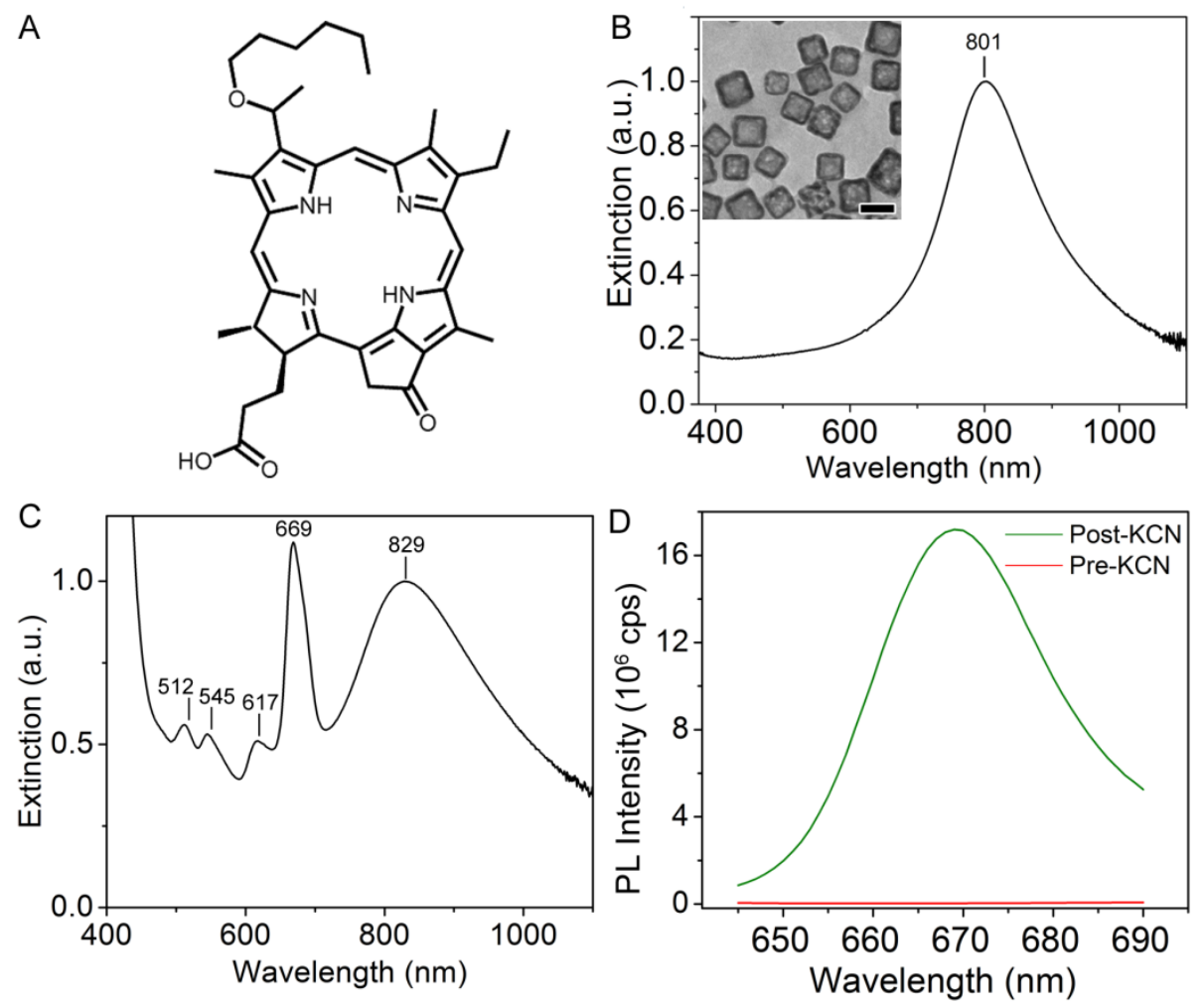

Figure 1. (A) Structure of HPPH. Normalized UV-vis-NIR spectra of AuNC (B) before and (C) after loading with HPPH. Inset shows TEM image of AuNCs with a $50 \mathrm{~nm}$ scale bar. (D) Fluorescence spectra of HPPH-loaded AuNCs, before (green) and after (red) KCN digestion of AuNCs.

The concentration of HPPH was determined by UV-vis spectroscopy based on its absorption coefficient in methanol $\varepsilon_{\mathrm{HPPH}, 669 \mathrm{~nm}} \approx 4.0 \times 10^{4} \mathrm{M}^{-1} \mathrm{~cm}^{-1}$. The incorporated HPPH per AuNC is readily changed by adjusting the ratio of $\mathrm{HPPH}$ to AuNC in the reaction. The loaded concentration of HPPH increased to a maximum on the order of $10^{5}$ HPPHs per AuNC. As the 
loading increased, the hydrodynamic diameter $\left(d_{H}\right)$ slightly increased from $98.7 \pm 0.9$ to $105.3 \pm$ $2.8 \mathrm{~nm}$ while the zeta potential decreased from $+6.4 \pm 0.5$ to $-26.3 \pm 0.8 \mathrm{mV}$ (Fig. 2A). The decrease of zeta potential is likely due to the increasing amount of negatively-charged HPPH in the complexes. The $d_{H}$ remained more or less constant, suggesting that HPPH was mostly entangled within the PEG layer on the nanocage surface rather than aggregating on top of the coating. Earlier theoretical work predicted that the addition of PEG to a solution of hematoporphyrin resulted in concentration-dependent porphyrin deaggregation and association with the PEG chains through strong interaction between the $-\mathrm{CH}_{2} \mathrm{CH}_{2}-$ region of the PEG chain and the porphyrin ring. ${ }^{24}$

The hydrophobic interaction between PEG and HPPH was further confirmed by studying the binding affinity. Increasing amounts of HPPH were introduced to phosphate buffer (10 mM, pH 7.4) with 0 or $135 \mathrm{mM} \mathrm{NaCl}$ at a AuNC-PEG concentration of $1 \mathrm{nM}$. After incubation overnight, the unbound HPPH concentration was determined by the absorbance at $669 \mathrm{~nm}$ upon removal of AuNCs by centrifugation. The bound HPPH $\left(L_{b}\right)$ was taken to be the difference between the total $\left(L_{t}\right)$ and the unbound HPPH $\left(L_{u}\right)$ concentration. No loss of HPPH was seen in the absence of AuNCs. A saturation binding isotherm (Fig. 2B) was developed, and Scatchard analysis results in a plot that is both hyperbolic and concave up, consistent with nonspecific binding between PEG and HPPH. ${ }^{34}$ The data were fitted to the equation $L_{b}=N \times L_{u} /\left(K_{d, n s}+L_{u}\right)$, where $K_{d, n s}$ is the dissociation constant for nonspecific binding and $N$ is a constant loosely related to the number of available binding sites. ${ }^{35}$ Based on the fitting, the $K_{d, n s}$ was determined to be $3.2 \times 10^{-}$ ${ }^{5} \mathrm{M}$ and $3.7 \times 10^{-5} \mathrm{M}$ in $0 \mathrm{mM}$ and $135 \mathrm{mM} \mathrm{NaCl}$, respectively, which is similar to simulation results on similar molecules; $;{ }^{24}$ the respective $\mathrm{N}$ values increased from $\sim 100$ to $\sim 180$. These data suggest that the ionic strength does not enhance binding between HPPH and PEG, but makes 
available additional binding sites and/or screens electrostatic repulsion, increasing the packing density of HPPH. The presence of aqueous ions significantly increased the available binding sites of PEG and HPPH due to a further exposure of $-\mathrm{CH}_{2} \mathrm{CH}_{2}-$ of PEG to the porphyrin ring of HPPH, which ultimately caused a near doubling the $\mathrm{N}$ value. Moreover, the loaded HPPH reached a maximum at $\sim 2.5 \times 10^{5} \mathrm{HPPHs} / \mathrm{AuNC}$, indicating that a finite space was available for loading within the PEG layer on the nanocage surface.
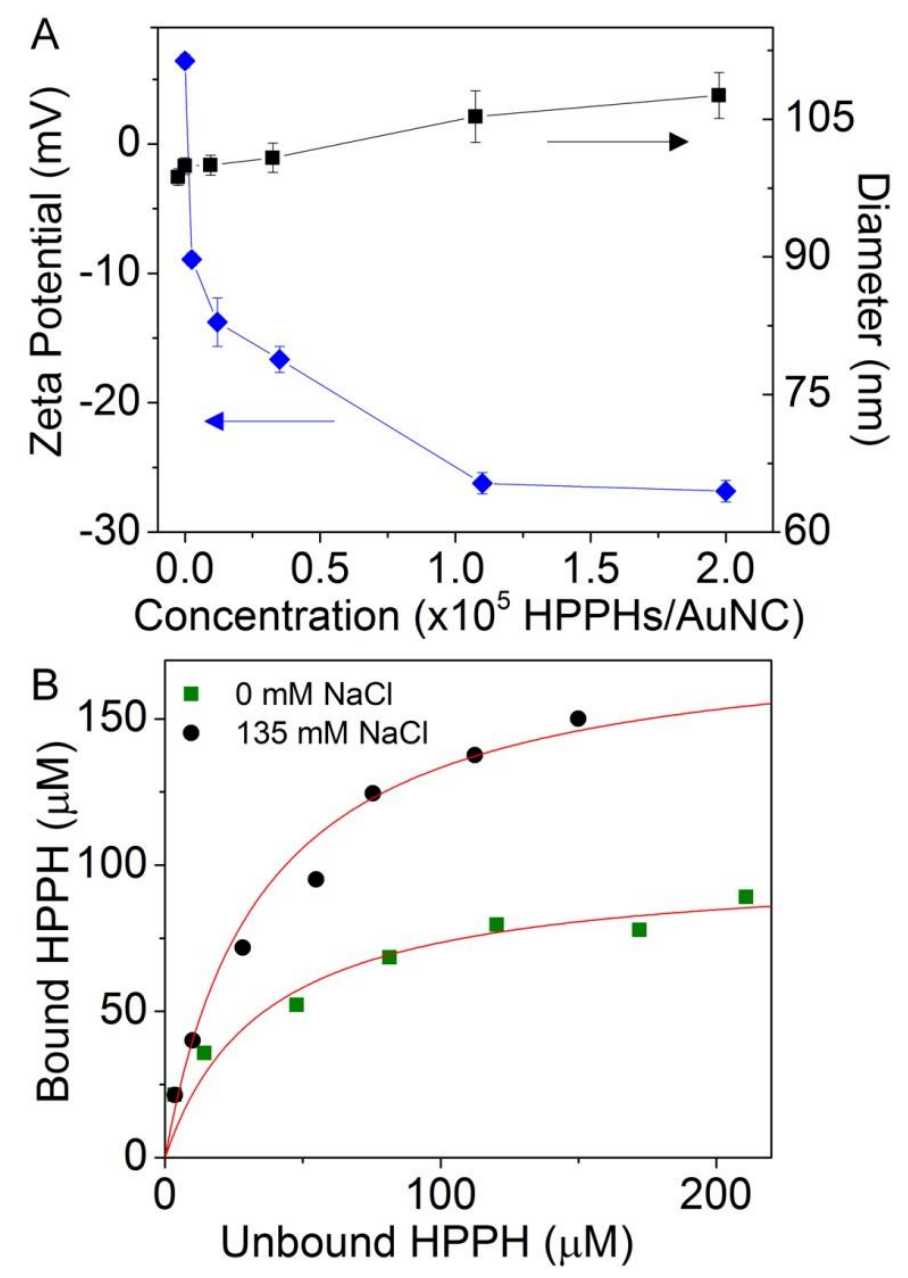

Figure 2. (A) Zeta potential (diamonds) and hydrodynamic diameter (squares) as measured by DLS for AuNC-HPPH at varying ratios of HPPH to AuNC. (B) Saturation binding isotherm between HPPH and the PEG coating on AuNC surface, AuNC concentration (1 nM) using $0 \mathrm{mM}$ (squares) or $135 \mathrm{mM}$ (circles) $\mathrm{NaCl}$ in $10 \mathrm{mM}$ phosphate buffer $(\mathrm{pH}=7.4)$. The red line indicates the fitting using the equation $L_{b}=N \times L_{u} /\left(K_{d, n s}+L_{u}\right)$. 
The fluorescent properties of HPPH inside the PEG layer on the AuNCs were examined by fluorescence lifetime measurements (Fig. 3A). Due to quenching, the quantum yields were very low, but a weak signal was present that was able to be picked up using the microscope setup. The HPPH lifetime ( $\tau_{\text {avg }}$ ) for AuNC-HPPH was measured to be 1.39 ns, comparable to that in PBS $(1.51 \mathrm{~ns})$ and appreciably shorter than in methanol (6.55 ns) or BSA (7.21 ns). Even when the loading concentration was reduced to $5 \times 10^{3} \mathrm{HPPHs}$ per AuNC, the lifetime increased only slightly to 2.69 ns. These data suggest that the HPPH molecules in AuNC-HPPH are in close proximity to each other, similar to the small aggregates that have been generated in PBS. ${ }^{36}$ The proximity of HPPH to the AuNC surface was further characterized by Raman spectroscopy (Fig. 3B). Raman spectra were acquired at excitation wavelength of $488 \mathrm{~nm}$ and the signal intensities were compared for different forms of HPPH at a concentration of $100 \mathrm{nM}$. The samples include free HPPH, AuNC-HPPH $\left(5 \times 10^{3}\right.$ HPPHs per AuNC), and AuNC-HPPH $\left(1 \times 10^{5}\right.$ HPPHs per AuNC). The peak at $1641 \mathrm{~cm}^{-1}$ was assigned to the weak Raman signal from water. The C-H peaks at $\sim 2950 \mathrm{~cm}^{-1}$ in these spectra likely originate from the PEG as the intensity does not change with the HPPH concentration. The broad, Gaussian region from 2000 to $2500 \mathrm{~cm}^{-1}$ (540$555 \mathrm{~nm}$ ) in the free HPPH was attributed to weak fluorescence of HPPH. The high loading concentration gave rise to a typical Raman peak at $1590 \mathrm{~cm}^{-1}$ that corresponds to the vibration of aromatic bonds in the macrocycle, ${ }^{37}$ suggesting that the pheophorbide ring is oriented somewhat perpendicularly to the surface of the metal. ${ }^{38}$ At low concentration, the Raman signature disappeared and was replaced by a broad fluorescence peak centered at $2340 \mathrm{~cm}^{-1}$. These data suggest that HPPH may be driven deeper in the coating and closer to the metal surface at higher concentrations, highlighting that HPPHs were densely packed within the PEG layer. 

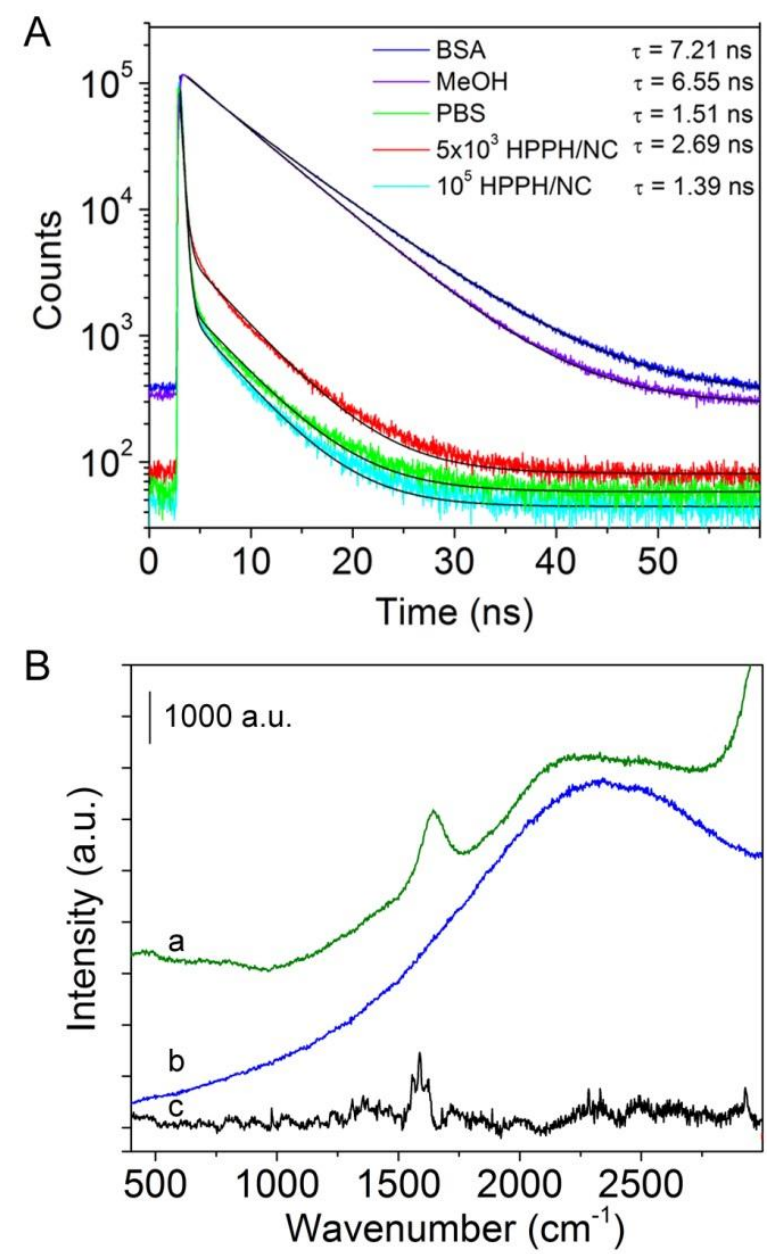

Figure 3. (A) Fluorescence lifetime measurements of HPPH under various conditions: dispersed in methanol (violet), 4\% w/w BSA solution (blue), PBS (green) and as AuNC-HPPH in PBS loaded at $5000 \mathrm{HPPHs} / \mathrm{AuNC}$ (red) and $10^{5} \mathrm{HPPHs} / \mathrm{AuNC}$ (cyan). (B) Raman spectra of $100 \mathrm{nM}$ HPPH under various conditions: (a) free HPPH, (b) AuNC-HPPH ( 5000 HPPHs/AuNC, 20 pM AuNC), and (c) AuNC-HPPH ( $10^{5}$ HPPHs/AuNC, 1 pM AuNC); spectra were acquired over 300 s using $488 \mathrm{~nm}$ excitation.

The release of the drug was monitored in a serum-mimetic environment to simulate intravenous injection (Fig. 4A) ${ }^{39}$ Fluorescence gradually recovered over $32 \mathrm{~h}$ when incubated in 4 wt $\%$ BSA $\left(\sim 600 \mu \mathrm{M}, 37^{\circ} \mathrm{C}, \mathrm{pH}=7.4\right) .{ }^{40}$ As a control, AuNC-HPPH was also incubated in PBS, and release was monitored using supernatant absorbance at various times compared to the maximum. It was found that $86.3 \pm 7.6 \%$ of $\mathrm{HPPH}$ had been unloaded in the BSA solution within $24 \mathrm{~h}$, but only $4.7 \pm 2.1 \%$ of the payload had unloaded in PBS. PEG monolayers are 
known to resist formation of a "hard" (nonremovable) protein corona, ${ }^{41,42}$ therefore the release is attributable to the formation of the "soft" protein corona, which entails rapid adsorption and desorption of serum proteins on the PEG surface. ${ }^{43,44}$ During this transient event, BSA presumably binds HPPH, which then desorbs with the protein. The $K_{d, n s}$ of PEG-HPPH complexes is on the order of $10^{-5} \mathrm{M}$, much larger than the typical $K_{d}$ values of BSA-porphyrin complexes $\left(\sim 10^{-9} \mathrm{M}\right),{ }^{45,46}$ ergo it would be energetically favorable for BSA to bind HPPH, releasing it from the PEG.

The PEG terminus was changed by conjugating AuNCs to either amine- or carboxylate-

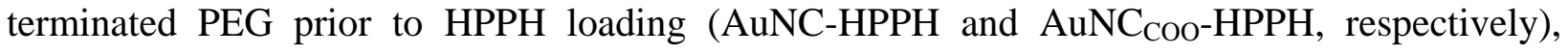
which was found to significantly affect the kinetics of HPPH release. Replacing cationic $\left(\mathrm{NH}_{3}{ }^{+}\right)$ with anionic $\left(\mathrm{COO}^{-}\right)$termini, resulted in slower unloading kinetics with only $50.1 \pm 6.8 \%$ release within $24 \mathrm{~h}$. Because the isoelectric point of BSA is 4.7 , it is negatively charged at $\mathrm{pH}$ of 7.4 , and should be repelled by the carboxylate terminus of the $\mathrm{PEG},{ }^{47}$ which in turn slows the unloading of HPPH. The release rate could be further retarded by cross-linking the termini of the PEGs on the surface (Fig. 4B). Amide coupling between the cross-linker, HOOC-PEG ${ }_{250}-\mathrm{COOH}$ (3-4 repeating units, M.W. $=250 \mathrm{Da}$ ), and the $\mathrm{NH}_{3}{ }^{+}$terminus of the PEG resulted in steric hindrance that blocked protein binding. Cross-linking with $\mathrm{PEG}_{250}$ resulted in a dramatic reduction of the HPPH release to only $31.1 \pm 8.7 \%$ within $32 \mathrm{~h}$ as compared to the release of $61.2 \pm 6.8 \%$ for the noncross-linked system. Similar steric hindrance was observed with blockcopolymeric micelles wherein crosslinking was prevented further from the water-polymer interface. $^{48}$ 

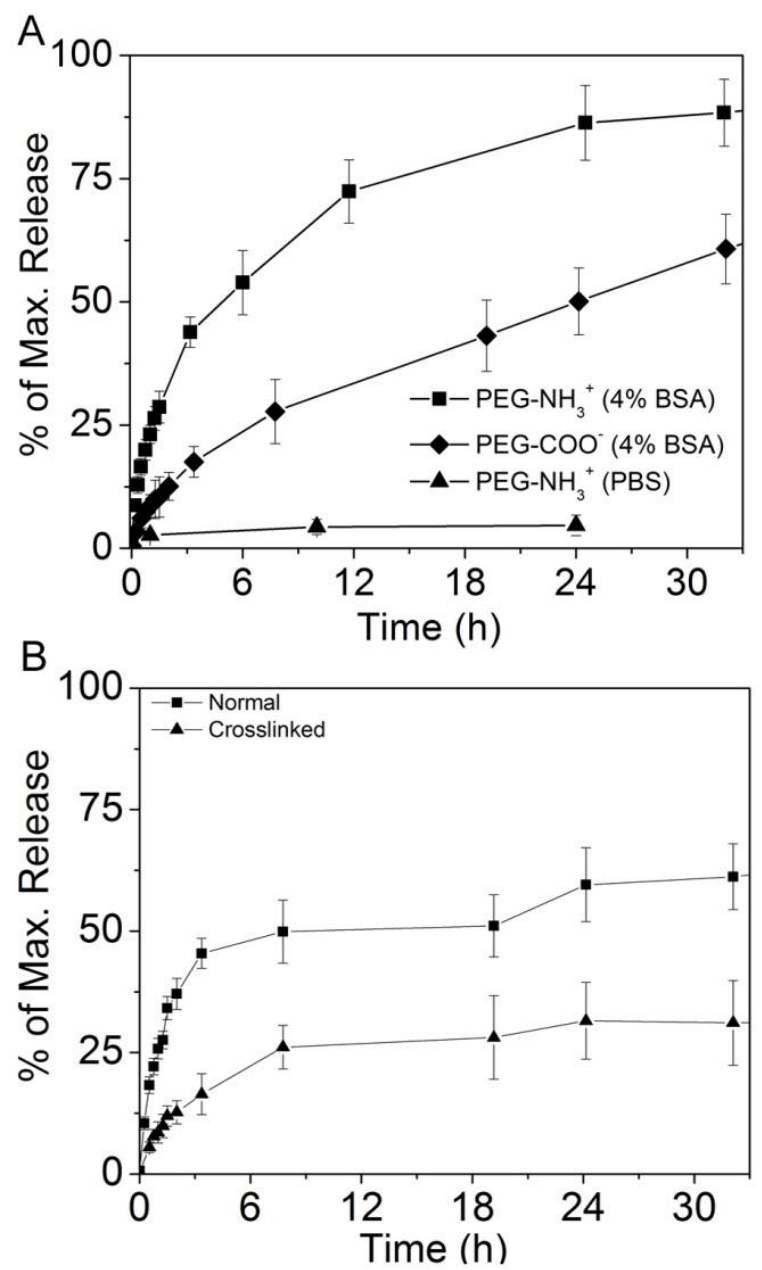

Figure 4. (A) Comparison of $\mathrm{HPPH}$ release kinetics at $\mathrm{pH}=7.4,37^{\circ} \mathrm{C}$ for AuNC-HPPH using PEG- $\mathrm{NH}_{3}{ }^{+}$(squares) and PEG-COO (diamonds) with $4 \%$ w/w BSA in PBS, and in only PBS (triangles). (B) Comparison of HPPH release kinetics from AuNC-HPPH without (squares) and with (triangles) cross-linking of the polymer terminus $\left(-\mathrm{NH}_{2}\right)$ by $\mathrm{HOOC}-\mathrm{PEG}_{250}-\mathrm{COOH}$. Maximum (100\%) values were determined by the recovered fluorescence signal after the AuNCs were digested by $\mathrm{KCN}$.

Ideally, AuNC-HPPH is anticipated to unload the HPPH in the tumor cell membrane for maximal photodynamic effect due to the short lifetime of singlet oxygen. Vesicles were prepared with a 3:1 mole ratio of DMPC and DMPG to mimic the composition of human cell membranes. Incorporation of HPPH in the vesicle membrane (1 mM lipid solution) leads to recovery of its fluorescence; therefore the release could be monitored similarly to BSA solutions. The release of HPPH to the vesicle solution was compared for free HPPH and AuNC-HPPH (Fig. 5). Similar to 
the BSA solution, $~ 90 \%$ of the HPPH unloaded from AuNC-HPPH after $24 \mathrm{~h}$. It is worth noting that unlike tumor cells, the vesicles were free in solution and could interact freely with suspended particles, artificially inflating the kinetics of the process. Interestingly, free HPPH did not reach its maximum signal until between 2 and $4 \mathrm{~h}$ incubation in the vesicle solution, whereas the BSA solution of free HPPH reached a maximum within $10 \mathrm{~min}$. These results imply that kinetics of HPPH adsorption is much more rapid for BSA than for the cell membrane. However, the ultimate release amount of HPPH from AuNC-HPPH is comparable for both systems, which suggests that diffusion is a major determining factor in release. As such, following intravenous injection HPPH would be less likely to be bound by BSA when delivered as AuNC-HPPH than free HPPH. Additionally, because of its accumulation in tumors, ${ }^{14}$ AuNC-HPPH would likely deliver a larger HPPH dose to the cell membrane itself.

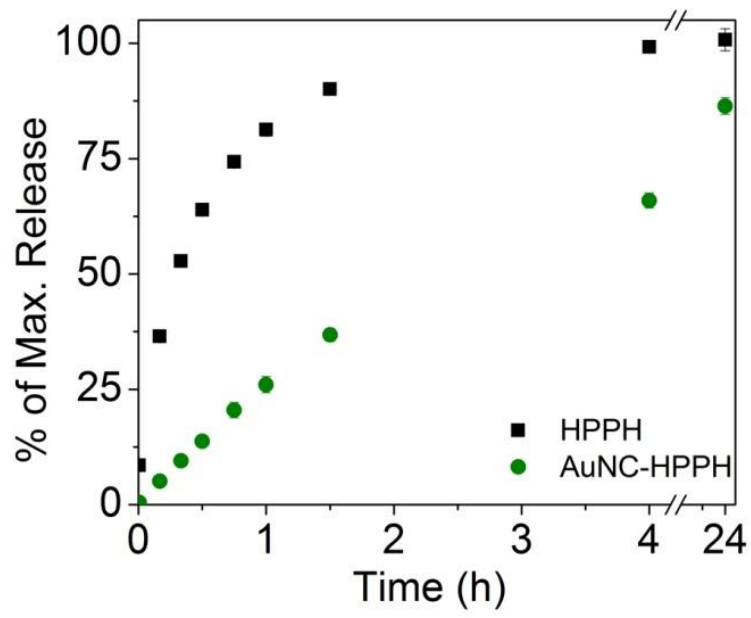

Figure 5. Kinetics of incorporation of HPPH into vesicles using free HPPH (squares) and AuNC-HPPH (circles).

The release could be further controlled with PT heating by the AuNCs (Fig. 6). Under NIR irradiation by a diode laser $\left(1 \mathrm{~W} / \mathrm{cm}^{2}\right.$ at $\left.808 \mathrm{~nm}\right)$ that overlaps with the LSPR, the solution temperature was held at $46{ }^{\circ} \mathrm{C}$ for $2 \mathrm{~h}^{49,50}$ In the dark, only $26.2 \pm 6.7 \%$ of the payload was 
released from the PEG layer after two hours. The slow release profile should provide enough time for the AuNC-HPPH to accumulate in the tumor prior to unloading, as demonstrated in our previous in vivo study. ${ }^{14}$ Irradiation led to localized heating, which presumably increased the fluidity of the PEG/HPPH layer, enabling HPPH to rapidly diffuse to the interface and interact with BSA. We speculate that this change might be the result of disruption of the hydration of the $\mathrm{PEG}^{51}$ and/or partial collapse of the PEG layer, ${ }^{52}$ increasing molecular motion due to thermal energy. A linear burst release $(69.2 \pm 5.0 \%)$ in the first 45 min was observed, after which the release proceeded gradually, only reaching $\sim 75 \%$ when the study terminated after two hours. The residual HPPH is unlikely to be trapped within the nanocage void because the loading capacity of AuNCs by diffusion to their interiors was 100 HPPHs/AuNC (Fig. S3), which accounted for only $0.1 \%$ of the total loaded HPPH. Thus, the remaining HPPH may have been buried too deeply within the PEG layer to have interacted with BSA for release.

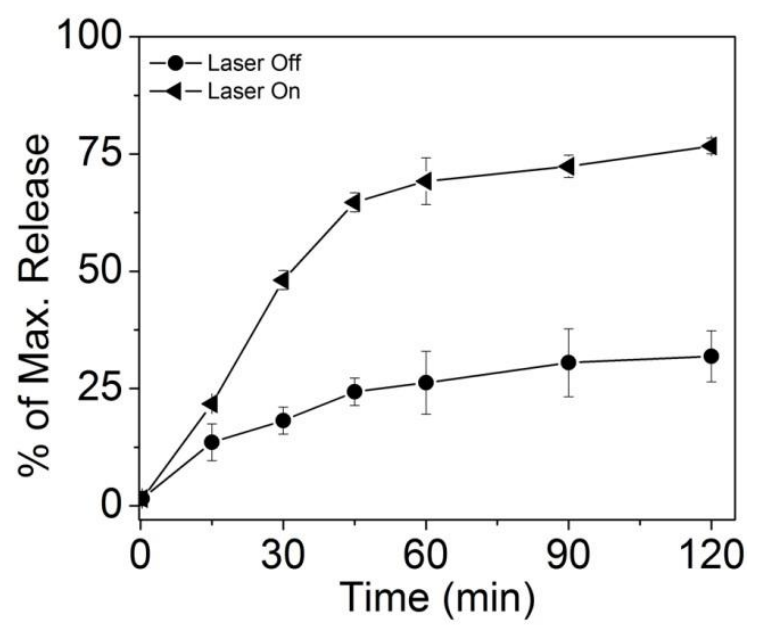

Figure 6. Comparison of HPPH release kinetics from AuNC-HPPH under irradiation by $808 \mathrm{~nm}$ diode laser at $1 \mathrm{~W} / \mathrm{cm}^{2}$ (triangles), and from AuNC-HPPH at $37{ }^{\circ} \mathrm{C}$ in the dark (circles). Maximum (100\%) values were determined by the recovered fluorescence signal after the AuNCs were digested by KCN. 
A finer measure of control was achieved using brief pulses of irradiation, and little release was observed during the dark time. The controlled release profiles are shown in Figure 7A, and the change in temperature of equimolar AuNCs in PBS is shown in Figure 7B. The suspension temperature rose from $37{ }^{\circ} \mathrm{C}$ to $46{ }^{\circ} \mathrm{C}$ during the 5 min irradiation period and returned to $37{ }^{\circ} \mathrm{C}$ during the 10 min dark time. $\mathrm{AuNC}_{\mathrm{COO}}-\mathrm{HPPH}$ and $\mathrm{AuNC}-\mathrm{HPPH}$ were separately incubated in BSA for 5 min prior to the first irradiation. During the first laser pulse roughly 20 and $30 \%$ of the respective payloads were released, and only a further $5 \%$ released in the dark time that followed. The second pulse resulted in 15 and $20 \%$ of the respective payloads being released, and the final pulse released $\sim 10 \%$ of the payload. Ultimately, $\sim 60 \%$ and $\sim 80 \%$ of the payload was released from $\mathrm{AuNC}_{\mathrm{COO}}-\mathrm{HPPH}$ and AuNC-HPPH, respectively. Faster release was observed for AuNC-HPPH than $\mathrm{AuNC}_{\mathrm{COO}}-\mathrm{HPPH}$, similar to the dark release (Fig. 7A). Both samples demonstrated similar capacity for on-demand release, though $\mathrm{AuNC}_{\mathrm{COO}}-\mathrm{HPPH}$ seemed to be more responsive to the irradiation. The slow release observed in the absence of irradiation suggests that little premature leakage would occur during transport from the injection site to the tumor, but the extensive, controllable release of the payload suggests that extensive unloading can be accomplished quickly within the tumor. Furthermore, release was achieved using photothermally therapeutic temperatures, suggesting that photothermal unloading could provide the benefits of both PT therapy and on-demand drug delivery. 


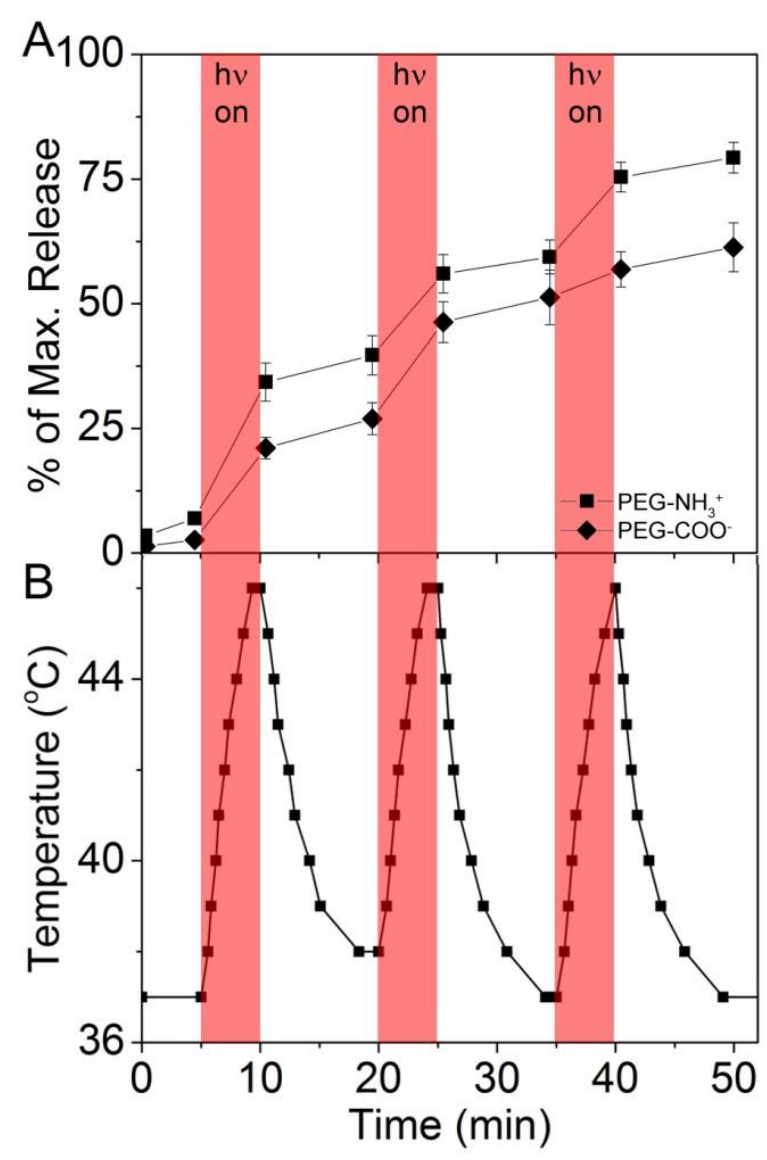

Figure 7. (A) On-demand release of HPPH from AuNC-HPPH as triggered by NIR irradiation at $808 \mathrm{~nm}\left(1 \mathrm{~W} / \mathrm{cm}^{2}\right)$. Maximum $(100 \%)$ values were determined by the recovered fluorescence signal after the AuNCs were digested by KCN. (B) Temperature change of sample solutions for cycles of $5 \mathrm{~min}$ irradiation and $10 \mathrm{~min}$ in the dark. Shaded regions indicate laser irradiation periods.

\section{CONCLUSION}

We have systematically investigated the use of PEG-coated AuNCs as a platform to deliver a photosensitizer namely HPPH by a number of methods including fluorescence intensity and lifetimes, Raman spectroscopy, DLS, and zeta potential measurements. HPPH was found to densely pack within the PEG coating, rather than aggregating at the surface. The loading capacity could be maximized to be $\sim 2 \times 10^{5} \mathrm{HPPHs} / \mathrm{AuNC}$, which is attributable to the hydrophobic interaction between HPPH and the PEG layer with a $\mathrm{K}_{\mathrm{d}}$ of $\sim 35 \mu \mathrm{M}$. Release of 
cargo from this delivery system was investigated in albumin-containing media to mimic in vivo delivery following intravenous injection and in vesicles to mimic unloading to the cell membrane. The release was found to be slightly faster for albumin, although the timeframes are comparable. This suggests that, in vivo, HPPH would be unloaded to both serum proteins and cell membranes. Changing the ionic character of the PEG terminus affected the release rate by modifying the interactions with dissolved proteins, and crosslinking the termini provides a steric block that prevents undesired unloading. On-demand release could be accomplished by using irradiation to induce the PT effect.

Release studies in a serum-mimetic environment and a suspension of cell membrane mimetic vesicles provide a general method to optimize drug delivery systems prior to costly in vitro and in vivo evaluation. In combination with our previous in vivo demonstration of AuNC-HPPH efficacy, ${ }^{14}$ this work has established fundamental understanding of the PEG-covered AuNC platform in the delivery of HPPH, which may help elucidate the fate of other non-covalent drugpolymer systems in vivo. The PEG-covered AuNC system integrated with stimuli-responsive cross-linkers can potentially serve as a multifunctional platform for controlled delivery of a wide variety of hydrophobic drugs. Further utilizing the optical properties of AuNC,,$^{50}$ these systems can achieve theranostics through diagnosis via AuNC contrast-enhanced molecular imaging and multi-modal treatment via photodynamic-, photothermal-, and chemo- therapies. 
Acknowledgement: This work was supported in part by the pilot project funds from the Arkansas Biosciences Institute, the National Institutes of Health (NIH P30 GM103450), the Ralph E. Powe Jr. Faculty Enhancement Award, and startup funds from the University of Arkansas, to J.C.; the financial support from Roswell Park Alliance to R.K.P; and an appointment to S.V.J. to the Summer Student Research Program at the National Center for Toxicological Research administered by the Oak Ridge Institute for Science and Education through an interagency agreement between the U.S. Department of Energy and the U.S. Food and Drug Administration. C.D.H. would like to thank the National Science Foundation (CHE1255440) for financial support. K.Y.R. thanks the support from Student Undergraduate Research Fellowship (SURF). The views presented in this article do not necessarily reflect those of the Food and Drug Administration. 


\section{References:}

1. Peer, D.; Karp, J. M.; Hong, S.; Farokhzad, O. C.; Margalit, R.; Langer, R. Nat Nano 2007, 2, 751-760.

2. Ghosh, P.; Han, G.; De, M.; Kim, C. K.; Rotello, V. M. Adv. Drug Del. Rev. 2008, 60, 1307-1315.

3. Gil, P. R.; Parak, W. J. ACS Nano 2008, 2, 2200-2205.

4. Farokhzad, O. C.; Langer, R. ACS Nano 2009, 3, 16-20.

5. Bardhan, R.; Lal, S.; Joshi, A.; Halas, N. J. Acc. Chem. Res. 2011, 44, 936-946.

6. Xia, Y.; Li, W.; Cobley, C. M.; Chen, J.; Xia, X.; Zhang, Q.; Yang, M.; Cho, E. C.; Brown, P. K. Acc. Chem. Res. 2011, 44, 914-924.

7. Dreaden, E. C.; Alkilany, A. M.; Huang, X.; Murphy, C. J.; El-Sayed, M. A. Chem. Soc. Rev. 2012, 41, 2740-2779.

8. Rana, S.; Bajaj, A.; Mout, R.; Rotello, V. M. Adv. Drug Del. Rev. 2012, 64, 200-216.

9. Xie, J.; Lee, S.; Chen, X. Adv. Drug Del. Rev. 2010, 62, 1064-1079.

10. Alkilany, A. M.; Lohse, S. E.; Murphy, C. J. Acc. Chem. Res. 2012, 46, 650-661.

11. Huang, J.; Jackson, K. S.; Murphy, C. J. Nano Lett. 2012, 12, 2982-2987.

12. Kim, C. K.; Ghosh, P.; Pagliuca, C.; Zhu, Z.-J.; Menichetti, S.; Rotello, V. M. J. Am. Chem. Soc. 2009, 131, 1360-1361.

13. Ren, F.; Bhana, S.; Norman, D. D.; Johnson, J.; Xu, L.; Baker, D. L.; Parrill, A. L.; Huang, X. Bioconjugate Chem. 2013, 24, 376-386.

14. Srivatsan, A.; Jenkins, S. V.; Jeon, M.; Wu, Z.; Kim, C.; Chen, J.; Pandey, R. K. Theranostics 2014, 4, 163-74.

15. Tam, N. C. M.; Scott, B. M. T.; Voicu, D.; Wilson, B. C.; Zheng, G. Bioconjugate Chem. 2010, 21, 2178-2182.

16. Sershen, S. R.; Westcott, S. L.; Halas, N. J.; West, J. L. J. Biomed. Mater. Res. 2000, 51, 293-298.

17. Knop, K.; Hoogenboom, R.; Fischer, D.; Schubert, U. S. Angew. Chem. Int. Ed. 2010, 49, 6288-6308.

18. Sun, G.; Hagooly, A.; Xu, J.; Nyström, A. M.; Li, Z.; Rossin, R.; Moore, D. A.; Wooley, K. L.; Welch, M. J. Biomacromolecules 2008, 9, 1997-2006.

19. Cheng, Y.; C. Samia, A.; Meyers, J. D.; Panagopoulos, I.; Fei, B.; Burda, C. J. Am. Chem. Soc. 2008, 130, 10643-10647.

20. Cheng, Y.; Samia, A. C.; Li, J.; Kenney, M. E.; Resnick, A.; Burda, C. Langmuir 2009, 26, 2248-2255.

21. Cheng, Y.; Meyers, J. D.; Broome, A.-M.; Kenney, M. E.; Basilion, J. P.; Burda, C. J. Am. Chem. Soc. 2011, 133, 2583-2591.

22. Ghosh, P.; Han, G.; De, M.; Kim, C. K.; Rotello, V. M. Advanced Drug Delivery Reviews 2008, 60, 1307-1315.

23. Ren, F.; Bhana, S.; Norman, D. D.; Johnson, J.; Xu, L.; Baker, D. L.; Parrill, A. L.; Huang, X. Bioconjugate Chemistry 2013, 24, 376-386.

24. Li, Y.-C.; Rissanen, S.; Stepniewski, M.; Cramariuc, O.; Róg, T.; Mirza, S.; Xhaard, H.; Wytrwal, M.; Kepczynski, M.; Bunker, A. J. Phys. Chem. B 2012, 116, 7334-7341.

25. Yavuz, M. S.; Cheng, Y.; Chen, J.; Cobley, C. M.; Zhang, Q.; Rycenga, M.; Xie, J.; Kim, C.; Song, K. H.; Schwartz, A. G.; Wang, L. V.; Xia, Y. Nat. Mater. 2009, 8, 935-939. 
26. Moon, G. D.; Choi, S.-W.; Cai, X.; Li, W.; Cho, E. C.; Jeong, U.; Wang, L. V.; Xia, Y. J. Am. Chem. Soc. 2011, 133, 4762-4765.

27. You, J.; Zhang, G.; Li, C. ACS Nano 2010, 4, 1033-1041.

28. Sershen, S. R.; Westcott, S. L.; Halas, N. J.; West, J. L. Journal of Biomedical Materials Research 2000, 51, 293-298.

29. Wilets, K. A.; Duyne, R. P. V. Ann. Rev. Phys. Chem. 2007, 58, $267-97$.

30. Yun, C. S.; Javier, A.; Jennings, T.; Fisher, M.; Hira, S.; Peterson, S.; Hopkins, B.; Reich, N. O.; Strouse, G. F. J. Am. Chem. Soc. 2005, 127, 3115-9.

31. Jennings, T. L.; Singh, M. P.; Strouse, G. F. J. Am. Chem. Soc. 2006, 128, 5462-7.

32. Xia, X.; Yang, M.; Oetjen, L. K.; Zhang, Y.; Li, Q.; Chen, J.; Xia, Y. Nanoscale 2011, 3, 950-3.

33. Biczok, L.; Valat, P.; Wintgens, V. Phys. Chem. Chem. Phys. 1999, 1, 4759-4766.

34. Mendel, C. M.; Mendel, D. B. Biochem J 1985, 228, 269-72.

35. van Zoelen, E. J. Biochem J 1989, 262, 549-56.

36. Baba, K.; Pudavar, H. E.; Roy, I.; Ohulchanskyy, T. Y.; Chen, Y.; Pandey, R. K.; Prasad, P. N. Mol. Pharm. 2007, 4, 289-297.

37. Cotton, T. M.; Schultz, S. G.; Duyne, R. P. V. J. Am. Chem. Soc. 1982, 104, 6528-32.

38. Gao, X.; Davies, J. P.; Weaver, M. J. J. Phys. Chem. 1990, 94, 6858-64.

39. Jenkins, S. V.; Qu, H.; Mudalige, T.; Ingle, T. M.; Wang, R.; Wang, F.; Howard, P. C.; Chen, J.; Zhang, Y. Biomaterials 2015, 51, 226-237.

40. Doweiko, J. P.; Nompleggi, D. J. J. Parenteral Enteral Nutrition 1991, 15, 207-11.

41. Harder, P.; Grunze, M.; Dahint, R.; Whitesides, G. M.; Laibinis, P. E. J. Phys. Chem. B 1998, 102, 426-436.

42. Ostuni, E.; Chapman, R. G.; Holmlin, R. E.; Takayama, S.; Whitesides, G. M. Langmuir 2001, 17, 5605-5620.

43. Walkey, C. D.; Olsen, J. B.; Guo, H.; Emili, A.; Chan, W. C. W. J. Am. Chem. Soc. 2011, 134, 2139-2147.

44. Walkey, C. D.; Chan, W. C. W. Chem. Soc. Rev. 2012, 41, 2780-2799.

45. Rotenberg, M.; Margalit, R. Biochem. J. 1985, 229, 197-203.

46. Mahammed, A.; Gray, H. B.; Weaver, J. J.; Sorasaenee, K.; Gross, Z. Bioconjugate Chem. 2004, 15, 738-746.

47. Hartvig, R. A.; van de Weert, M.; Østergaard, J.; Jorgensen, L.; Jensen, H. Langmuir 2011, 27, 2634-2643.

48. Sun, G.; Lee, N. S.; Neumann, W. L.; Freskos, J. N.; Shieh, J. J.; Dorshow, R. B.; Wooley, K. L. Soft Matter 2009, 5, 3422-3429.

49. Chen, J.; Glaus, C.; Laforest, R.; Zhang, Q.; Yang, M.; Gidding, M.; Welch, M. J.; Xia, Y. Small 2010, 6, 811-817.

50. Chen, J.; Yang, M.; Zhang, Q.; Cho, E. C.; Cobley, C. M.; Kim, C.; Glaus, C.; Wang, L. V.; Welch, M. J.; Xia, Y. Adv. Funct. Mater. 2010, 20, 3684-3694.

51. Hey, M. J.; Ilett, S. M.; Davidson, G. J. Chem. Soc., Faraday Trans. 1995, 91, 3897-3900.

52. Karlstroem, G. J. Phys. Chem. 1985, 89, 4962-4964. 


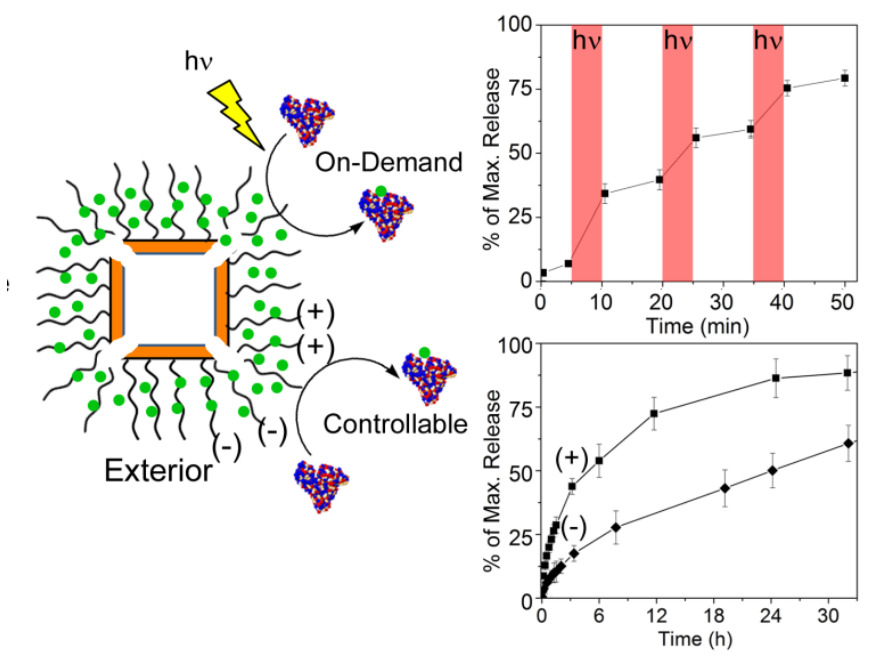

Graphical Abstract 